\title{
Venus Flytrap Seedlings Show Growth-Related Prey Size Specificity
}

\author{
Christopher R. Hatcher and Adam G. Hart \\ School of Natural and Social Sciences, University of Gloucestershire, Francis Close Hall, Cheltenham, GL50 4AZ, UK \\ Correspondence should be addressed to Christopher R. Hatcher; chris.hatcher@btinternet.com
}

Received 13 December 2013; Revised 28 January 2014; Accepted 4 February 2014; Published 18 March 2014

Academic Editor: Ram C. Sihag

Copyright ( 2014 C. R. Hatcher and A. G. Hart. This is an open access article distributed under the Creative Commons Attribution License, which permits unrestricted use, distribution, and reproduction in any medium, provided the original work is properly cited.

\begin{abstract}
Venus flytrap (Dionaea muscipula) has had a conservation status of vulnerable since the 1970s. Little research has focussed on the ecology and even less has examined its juvenile stages. For the first time, reliance on invertebrate prey for growth was assessed in seedling Venus flytrap by systematic elimination of invertebrates from the growing environment. Prey were experimentally removed from a subset of Venus flytrap seedlings within a laboratory environment. The amount of growth was measured by measuring trap midrib length as a function of overall growth as well as prey spectrum. There was significantly lower growth in prey-eliminated plants than those utilising prey. This finding, although initially unsurprising, is actually contrary to the consensus that seedlings (traps $<5 \mathrm{~mm}$ ) do not catch prey. Furthermore, flytrap was shown to have prey specificity at its different growth stages; the dominant prey size for seedlings did not trigger mature traps. Seedlings are capturing and utilising prey for nutrients to increase their overall trap size. These novel findings show Venus flytrap to have a much more complex evolutionary ecology than previously thought.
\end{abstract}

\section{Introduction}

Charles Darwin in a letter to Asa Gray [1] wrote "I care more for Drosera (now Dionaea) than the origin of species ... it is a wonderful plant, or rather a most sagacious animal. I will stick up for Drosera to the day of my death."

That speaks of the ecological and evolutionary significance of this plant. No other plant holds such a high reputation in its specialism to carnivory than Venus flytrap, Dionaea muscipula, and the fascination Darwin had for this plant is shared by modern scientists. In great part, this is because of the rapid closure of its trap (e.g., [2-4]). The closure of $D$. muscipula traps, each formed by the hinging of two specialised leaves, is one of the fastest movements in the plant kingdom [5]. Though there is an abundance of information on the physiology and mechanics of Venus flytrap's trap closure [6-9], Venus flytrap ecology is far less understood $[10,11]$. While the abundance of information on trap physiology is of great interest in understanding plant movement, it gives little benefit in relation to preserving this already vulnerable species $[12,13]$. There are very few published studies that focus on Venus flytrap's overall ecology and life cycle and, to our knowledge, no studies focus on seedling establishment from seed in respect to prey utilisation $[11,14,15]$. Consequently, little if anything is known about nutrient uptake, responses to environmental factors such as light, soil type, and moisture, and the prey requirements of seedling stages.

The few ecological studies that have been carried out indicate that, although Venus flytrap is highly adapted to its habitat (maximum nitrogen acquisition can be $92 \%$ from insects [16]), they are still outcompeted by other plants over time [17]. Venus flytrap longevity in habitats can be maintained by controlling habitat succession. Preventing progression to dense woody vegetation significantly reduces competition for light and space and benefits Venus flytrap $[18,19]$. Indeed, the disturbance provided by a frequent fire regime can increase Venus flytrap fitness by maintaining open vegetation with higher light levels [19]. However, closer consideration of the emergence and success of seedlings may prove more insightful, given the importance of these potentially plentiful juvenile stages in establishing an adult 


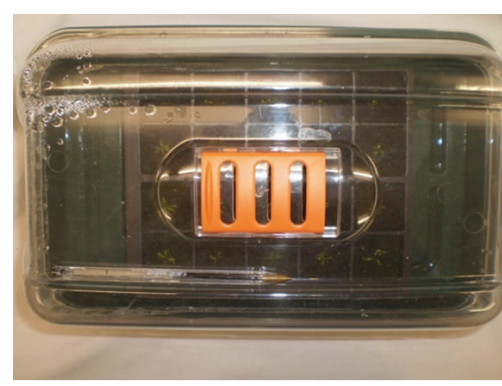

(a)

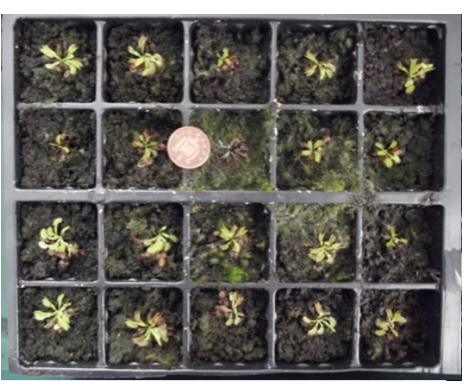

(b)

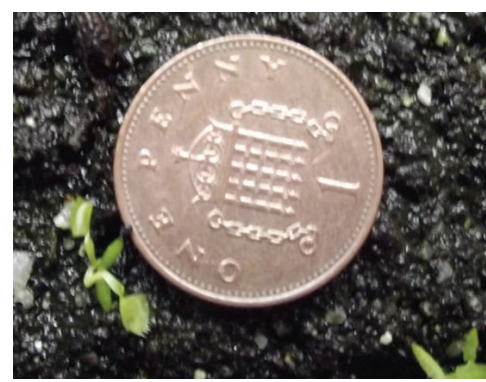

(c)

Figure 1: Propagation tank (a). Plant cell trays (b). Juvenile Venus flytrap (c) (scale 1 GB penny, diameter 20.3 mm).

population. More substantial empirical studies on all life stages and prey spectra are necessary to develop an understanding of Venus flytrap ecology and its conservation [20].

Many studies of carnivorous plant have examined the photosynthetic rates of leaves adapted for carnivory and considered the evolutionary switch between noncarnivory and carnivory [14, 21-24]. However, there have been far fewer comprehensive studies of carnivory based on growth stage or the prey capture capabilities of juvenile leaves [21, $25,26]$. Moreover there has not been a study primarily on Venus flytrap seedlings capturing prey and the effect of their nutrient acquisition in the transition to maturity. Here, the growth of juvenile Venus flytrap plants that have access to, or are excluded from, invertebrate prey was studied. As well as providing novel information on the reliance of Venus flytrap juvenile stages on prey capture, the spectrum of prey captured by the smaller plants was also examined.

\section{Materials and Methods}

2.1. Study Species. Dionaea is a rosette forming plant with green leaves that form well-characterised prey catching traps, enhanced by red pigment within the trap lobes [27]. The leaf petioles are the main sites of photosynthesis and succulent hypocotyl stores nutrients. The root system has no mycorrhizal associations [15]. The apex forms leaves, which have functional traps once they have completely unfolded [27].

2.2. Seedling Collection and Preparation. Two commercially purchased mature Dionaea muscipula plants flowering simultaneously were hand-pollinated to produce 120 seeds. These seeds were placed in peat moss and kept damp until germination. Once cotyledons had progressed into the first stage of trap formation, the plants were transplanted into fresh sterilised Irish peat moss from a commercial supplier (these were halved randomly into two groups referred to as "Related 1 " and "Related 2"). An additional 60 flytraps of various cultivars in the second rosette stage were collected from a commercial plant nursery, referred to as "SWCP." These plants were included to introduce diversity in juvenile plant age to allow the effect of prey on growth to be ascertained across different juvenile rosette stages of growth in the study period.
2.3. Experimental Design and Procedure. Seedlings were divided into $4 \times 5$ configured free-draining cell trays $(20$ cells per tray, each cell $4 \mathrm{~cm} \times 4 \mathrm{~cm}$ ), which were then placed into individual identical propagation tanks without drainage holes. These were placed at large windows in a thermoregulated laboratory and their order along the windows changed daily. Peat moss in the tray cells was sterilised using an autoclave at $90^{\circ} \mathrm{C}$ for 15 minutes. The tanks were covered with clear plastic lids with a closable vent (Figure 1). The plants had a natural light/dark cycle in a four-month period between August and October in Cheltenham, UK $\left(51.9^{\circ} \mathrm{N}\right.$, $\left.2.1^{\circ} \mathrm{W}\right)$, and temperature was regulated to keep it above $18^{\circ} \mathrm{C}$ to prevent dormancy. The temperature inside the tanks varied between $18^{\circ} \mathrm{C}$ and $35^{\circ} \mathrm{C}$. Soil acidity before and after sterilisation remained at an acceptable level between $\mathrm{pH}$ 5.2-5.5 [28] and there were regular visual inspections for organisms repopulating the soil. If contamination occurred, the medium could be replaced with sterilised peat moss; but in practice, this problem did not arise. The seedlings were bottom-watered once a day with deionised water to maintain a continuously moist environment for the trays. The soil was checked daily and any other plants rooted in the soil were removed as soon as they were visible. Green algal growth on the topsoil was also removed when it appeared.

Unsterilised peat had numerous springtails and other small invertebrates free-living within it. Sterilising the peat killed this fauna. The appropriate tanks ("prey present" tanks) were populated with approximately 200 springtails. Of nine tanks, each containing 20 plants, three tanks ( 60 plants; 1 tank of SWCP and 2 tanks of related plants) had the vent shut and had springtails present and therefore plants within these tanks could not use aerial insects but could still acquire prey from the soil ("Soil"; $N=60$ ). Three tanks had an open vent with a population of springtails and thus could acquire aerial prey as well as springtails ("Soil and Aerial"; $N=60 ; 1$ tank of SWCP and 2 tanks of related plants). The final three tanks had neither introduced springtails nor an open vent and thus had no prey available to the plants within this treatment ("No Prey"; $N=60 ; 1$ tank of SWCP and 2 tanks of related plants).

2.4. Plant Analysis. Seedlings were regularly checked and the number of leaves present was counted. Leaves counted were fully formed and healthy (green). Where traps were present, 


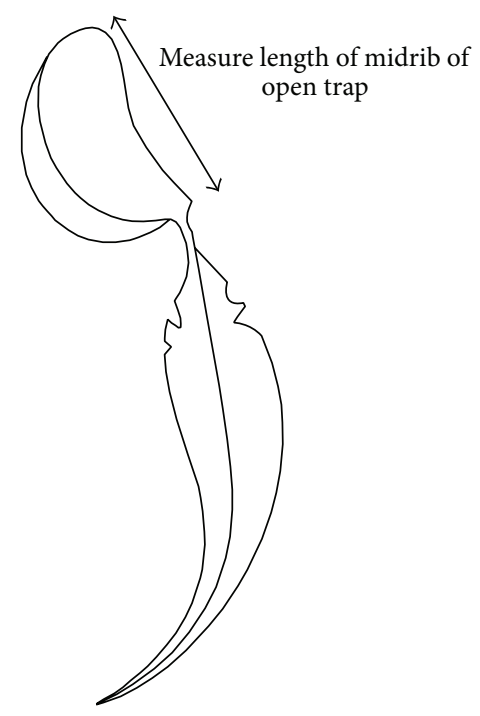

Figure 2: Trap length was measured with callipers by measuring the midrib of an open trap as indicated.

trap size was measured using digital Fisher Scientific callipers (model: FB70250 calibrated, compliant with ISO/IEC 17025 $\pm 0.005 \mathrm{~mm}$ ). In addition, any closed traps were recorded giving an indicator of trap function and frequency and the ability for single traps to open and close repeatedly, though prey capture success rates were not known. Recording trap closure also monitors the sterility of the soil; if a plant in the "No Prey" treatment has many closed traps and signs of exoskeletons, contamination would be indicated. Traps that lost photosynthetic pigment and subsequent trap function were not counted in total leaf number, as senescence of leaves is part of the process of forming new leaves in rosette forming plants. Growth and development of juvenile Venus flytrap was measured as the change from the mean initial trap midrib length of the largest five traps to the mean trap midrib length of the largest five traps at the end of the four-month growth period (Figure 2). This indicates the change in overall growth, rather than inadvertently measuring the same traps, which do not change in size in rosette forming plants [27]. To test whether Venus flytrap has growth-related prey size specificity, living springtails were placed into the lobes of large traps and observed until either the trap was triggered or the springtail left the lobe. This was repeated 50 times on two mature plants on five large traps. At the end of this test, houseflies were placed into each trap to confirm that the mechanism was functional.

2.5. Statistical Analysis. The interaction of the presence or absence of prey on the growth of juvenile Venus flytrap was assessed using a linear mixed effects model (LMER) with treatment ("Soil," "Aerial \& Soil," and "No Prey") and groups (SWCP, Related 1, or Related 2) as a fixed effect and tank as a random effect. The significance of fixed effects was tested using likelihood ratio tests, which follow chi-square distributions. Thus the results of the model are presented in this paper with significance assessed by the magnitude of chi-squared statistics $(P<0.05)$. All statistical analyses were carried out with the software package $R$ (version 3.0.1 [29]).

\section{Results}

3.1. Observations. In this study it was found that seedlings form their first traps after initial cotyledons that are functional in insect capturing after just a few weeks. Juvenile flytraps varying in size between $1.16 \mathrm{~mm}$ and $2 \mathrm{~mm}$ almost exclusively captured springtails (Collembola) found within the soil. Larger traps $(>2 \mathrm{~mm}-5.93 \mathrm{~mm}$ ) caught springtails and small flies in those that had access to winged insects. Apparently fully functional traps appeared in the first rosette stage, with traps $<2 \mathrm{~mm}$ able to open and close from mechanical stimulation (Figure 1(c)). Of plants able to capture prey ("Soil" and "Soil and Aerial"), 146/178 plants were recorded to have at least one trap on a plant close and reopen three times or more (82\%). Function as a trap ceased after repeated closing and the leaf remained as only a (assumed) photosynthetic organ. Across the duration of the experiment, the proportion of traps closed per plant was as follows: "Soil" $=0.30$; "Soil and Aerial" = 0.33; "Absent" $=0.08$. Prey was observed being caught during 2-hour monitoring periods fortnightly and exoskeletons were regularly seen within trap lobes. In the tanks able to acquire aerial insects, flies (Diptera) were occasionally seen within the tank and their exoskeletons were observed in traps ten times over the duration of the experiment during the 2-hour observation periods. Observations from this study comparing mature and juvenile Venus flytraps confirm that, at a certain size, the sensory trigger hairs are not excited sufficiently by smaller prey (such as springtails) introduced into the trap by hand. Traps are only triggered by larger prey that can produce a sufficient displacement of the mechanosensory hairs.

3.2. Juvenile Plant Growth. Of the 180 plants, only two perished (both from SWCP tank with vent shut and soil prey available) and these were not included in data analysis $(1.11 \%$ mortality). The range of initial trap size was $1.16-5.93 \mathrm{~mm}$ with a mean length of $2.72 \mathrm{~mm}(\mathrm{SD}=0.81)$ (Table 1). Final trap size ranged from 1.88 to $6.18 \mathrm{~mm}$ with a mean length of $3.14 \mathrm{~mm}(\mathrm{SD}=0.99)$. There were significant differences between the growth of plants with prey present in "Soil" and "No Prey" (LMER: $X_{5}^{2}=12.444, P<0.001$, Figure 3). There was also a significant difference between the growth of plants present in "Soil and Aerial" and "No Prey" (LMER: $X_{5}^{2}=9.302, P=0.002$, Figure 3 ). The two different types of treatment with prey were also significantly different from each other (LMER: $X_{5}^{2}=4.19, P=0.041$, Figure 3 ). Analysis showed that although size of traps varied from treatment, the total number of traps was not significantly different (LMER: $X_{5}^{2}=3.1235, P=0.201$, Table 2).

\section{Discussion}

This study presents novel insight into the relationship between carnivory and growth in Venus flytrap in early life stages. Even in juvenile stages, the reliance of Venus flytrap on 


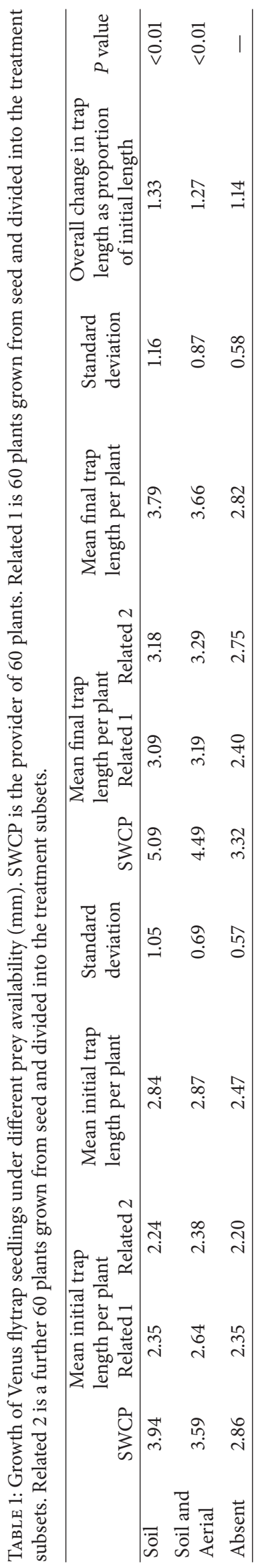




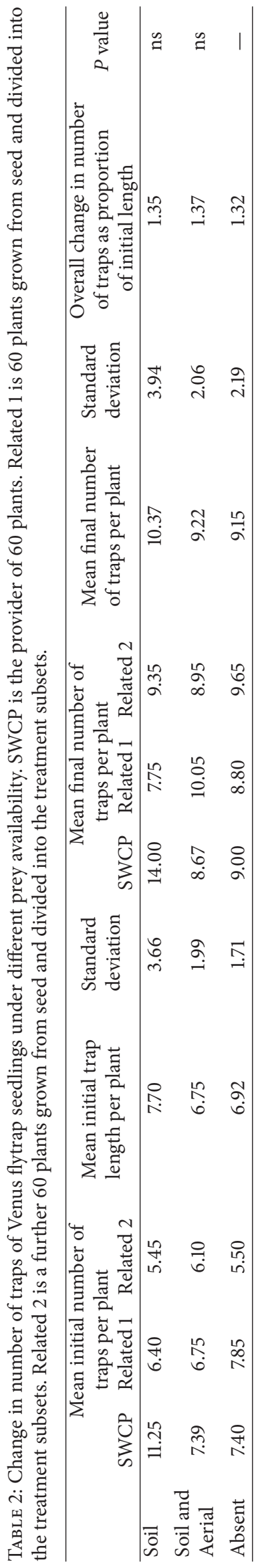




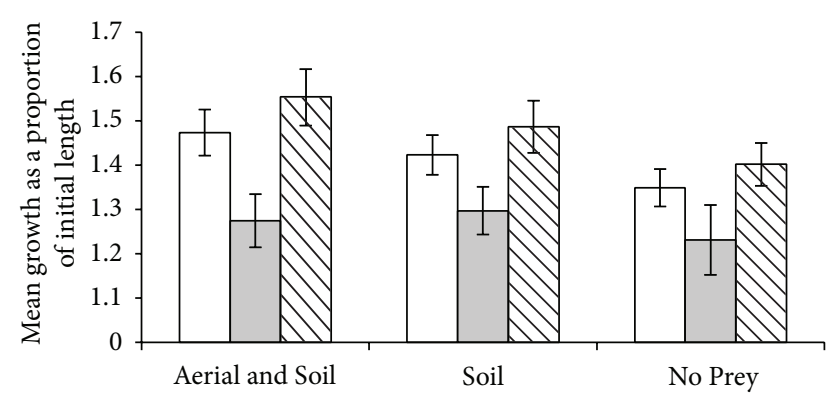

FIGURE 3: Mean growth from each treatment as a proportional increase in trap length as a function of growth (error bars are SE). White bars are all plants used in the experiment $N=178$ plants; grey bars are plants from SWCP (a carnivorous plant supplier) $N=$ 58 ; hatched bars are plants propagated from two mature plants and grown from seed $N=120$.

carnivory to obtain nutrients (especially nitrogen) for growth is considerable (Figure 3, $P<0.001$ ) (also [16]). The study also found that prey spectrum usage varied depending on trap size. Small traps cannot catch large insects and, more surprisingly, small organisms do not trigger the sensitive hairs on large traps. In terms of growth, it is interesting to confirm that only lobe and subsequent petiole length are affected, not the numbers of traps present [17]. Consequently, the evolutionary response to catching prey is to direct resources into making larger traps rather than increasing the probability of prey capture directly by increasing the number of traps (although the rosette growth form may limit the number of traps produced). However, increasing the size of a trap potentially increases the total prey mass available to the plant as, assuming larger prey is present, it incorporates larger organisms into its total possible prey capture spectrum. The benefit of catching a large organism is greater than catching a smaller prey, both in total nutrient quantity and in ratio of unusable material to nutrients [30].

The evidence found here regarding repeated trap functionality contradicts the findings cited by Juniper et al. [31] and Schulze et al. [27]. The findings from this study serve to underline that Dionaea is an even more highly specialised organism than previously thought and that it is adapted throughout its life history to suit nutrient poor soils and optimise net nutrient intake from prey. The evolution of Dionaea traps appears to have been driven by prey size. They have a common ancestor with modern Drosera. Presumably they were able to differentiate on the basis of catching larger prey, which led to the development of the snap trap [32]. This study suggests ontological variation in prey size capture. Collembola caught in traps $<3 \mathrm{~mm}$ did not trigger mature traps. Though this is not conclusive of the trapping habits in situ, it would seem that nutrient acquisition throughout development is a strong selective pressure in Dionaea life history.

Burning of Venus flytrap habitat is a standard management procedure currently and has shown to benefit Venus flytrap on the long term if the interburning intervals are appropriate $[10,18]$. However, findings from this study may indicate a necessity to alter the regime to promote seedling survival to maturity as a priority. Over time this would act to increase total population of Venus flytrap and provide a more diverse age range. Moreover, genetic diversity will also be increased, as plants arising from germinated seeds become a larger fraction of the population rather than those arising from budding.

Carnivory in Venus flytrap commences from the production of the first trap, which is the first leaf produced after cotyledons. The sizes of the first traps are less than $2 \mathrm{~mm}$ in length and, in this study, were effective in trapping small prey, notably springtails. At a certain trap size, springtails do not trigger sensory hairs and are removed from the prey spectrum, allowing for only larger, more beneficial prey to be acquired. It can be concluded that the ability for traps to be functional for multiple times provides a massive benefit, with renewing functionality being presumably cheaper than investing in "one trap per prey capture" as was thought to be the case by Juniper et al. [31] and Schulze et al. [27]. Moreover, when functionality ceases, rather than the leaf dying as previously stated in literature [27], the leaf continues to survive as a noncapturing leaf. Given the cost of producing new leaves and the plants requirement for carbohydrate as well as nitrogen, there is much to gain from maintaining a leaf until the next rosette stage.

\section{Conclusions}

Venus flytrap is classified as vulnerable in IUCN's Red List [12]. However, the census was carried out over a decade ago and the status is likely to have been exacerbated due to habitat destruction [11]. Seedling growth is a crucial aspect of flytrap ecology, as growth at this stage is slow due to the small size of prey they can acquire. The findings presented here suggest that focus on community ecology could improve conditions in which Venus flytrap could thrive. Increasing populations of invertebrates across the prey spectrum would increase seedling survival as well as increasing the probability of mature plants being able to reproduce without the risk of depleting resources beyond recovery. These two growth stages of venus flytrap are their most vulnerable due to; seedling's reliance on small prey and thus low levels of nitrogen, and resources required for flower production, which can also reduce the number of traps on mature plants [27]. Further work in situ would provide much needed insight into the natural diet of Venus flytrap seedlings. Though we have empirically shown a reliance on prey ex situ, understanding prey composition within their natural habitat is crucial.

Research into prey dynamics across different growth stages could also provide further clarity to the evolution of carnivory. Ellison and Gotelli [24] devote a section of their paper to the extent of which Dionaea selectively catch larger prey. Of the data they collated, the mean prey size was around $8 \mathrm{~mm}$ and the smallest size of prey caught, of which was deemed an outlier, was $4 \mathrm{~mm}$. Here the prey available to the Venus flytrap seedlings were all much smaller than this size. This would suggest adaptation to catching larger prey as trap size increases. Selectivity of prey size has been confirmed in Dionaea to an extent. Prey that trigger the mechanism have 
the opportunity to escape through the "bars" of an initially closed trap, preventing wasting resources on smaller prey. This study suggests further adaptation to prey size selectivity in that very small prey is utilised by seedlings but did not trigger traps on mature plants. Further examination in situ to confirm natural prey size selectivity is recommended. If, indeed, very small prey does not trigger mature traps, other interesting questions arise.

Whether this is due to a higher threshold of mechanical stimulation as a result of adaptation to catch larger, more profitable prey without wasting resources on smaller prey or simply a byproduct of a larger structure requiring more force to trigger the mechanism should be tested.

Here we have shown Venus flytrap ecology to be far more complex than previously documented and have empirically shown a reliance on prey from a very early stage in growth, giving insight into future research and conservation direction.

\section{Conflict of Interests}

The authors declare that they have no conflict of interests.

\section{Acknowledgments}

The authors thank Hannah Stubbs for her laboratory support and the Southwest Carnivorous Plants shop for supplying a number of Venus flytraps. Data and findings in this paper are not under consideration for publication anywhere else and all authors have seen and agreed to the submitted version of the paper.

\section{References}

[1] F. Jones, "The most wonderful plant in the world. With some unpublished correspondence of Charles Darwin," Natural History, vol. 23, no. 6, pp. 589-596, 1923.

[2] Y. Forterre, J. M. Skotheim, J. Dumals, and L. Mahadevan, "How the Venus flytrap snaps," Nature, vol. 433, no. 7024, pp. 421-425, 2005.

[3] R. Yang, S. C. Lenaghan, M. Zhang, and L. Xia, "A mathematical model on the closing and opening mechanism for venus flytrap," Plant Signaling and Behavior, vol. 5, no. 8, pp. 968-978, 2010.

[4] M. Escalante-Pérez, E. Krol, A. Stange et al., "A special pair of phytohormones controls excitability, slow closure, and external stomach formation in the Venus flytrap," Proceedings of the National Academy of Sciences of the United States of America, vol. 108, no. 37, pp. 15492-15497, 2011.

[5] V. Markin, A. Volkov, and E. Jovanov, "Active movements in plants, mechanism of trap closure by Dionaea muscipula Ellis," Plant Signaling \& Behavior, vol. 3, no. 10, pp. 778-783, 2008.

[6] E. Krol, H. Dziubinska, M. Stolarz, and K. Trebacz, "Effects of ion channel inhibitors on cold- and electrically-induced action potentials in Dionaea muscipula," Biologia Plantarum, vol. 50, no. 3, pp. 411-416, 2006.

[7] A. G. Volkov, T. Adesina, and E. Jovanov, "Closing of venus flytrap by electrical stimulation of motor cells," Plant Signaling and Behavior, vol. 2, no. 3, pp. 139-145, 2007.
[8] A. G. Volkov, H. Carrell, and V. S. Markin, "Biologically closed electrical circuits in Venus flytrap," Plant Physiology, vol. 149, no. 4, pp. 1661-1667, 2009.

[9] A. G. Volkov, V. A. Murphy, J. I. Clemmons, M. J. Curley, and V. S. Markin, "Energetics and forces of the Dionaea muscipula trap closing," Journal of Plant Physiology, vol. 169, no. 1, pp. 5564, 2012.

[10] J. O. Luken, "Habitats of Dionaea muscipula (Venus' fly trap), Droseraceae, associated with Carolina bays," Southeastern Naturalist, vol. 4, no. 4, pp. 573-584, 2005.

[11] J. Luken, "Long-term outcomes of Venus flytrap (Dionaea muscipula) establishment," Restoration Ecology, vol. 20, no. 6, pp. 669-670, 2012.

[12] D. Schnell, P. Catling, G. Folkerts, C. Frost, and R. Gardner, Dionaea muscipula IUCN Red List of Threatened Species, Version 2011.2, 2000.

[13] J. O. Luken, “Dionaea muscipula (Venus flytrap) establishment, release, and response of associated species in mowed patches on the rims of Carolina bays," Restoration Ecology, vol. 13, no. 4, pp. 678-684, 2005.

[14] A. M. Ellison and N. J. Gotelli, "Evolutionary ecology of carnivorous plants," Trends in Ecology and Evolution, vol. 16, no. 11, pp. 623-629, 2001.

[15] P. Roberts and J. Oosting, "Responses of Venus flytrap (Dionaea muscipula) to factors involved in its endemism," Ecological Monographs, vol. 28, pp. 193-218, 1958.

[16] W. Schulze, E. D. Schulze, J. S. Pate, and A. N. Gillison, "The nitrogen supply from soils and insects during growth of the pitcher plants Nepenthes mirabilis, Cephalotus follicularis and Darlingtonia californica," Oecologia, vol. 112, no. 4, pp. 464-471, 1997.

[17] J. O. Luken, "Performance of Dionaea muscipula as influenced by developing vegetation," Journal of the Torrey Botanical Society, vol. 134, no. 1, pp. 45-52, 2007.

[18] D. Schnell, Carnivorous Plants of the United States and Canada, Timber Press, Portland, Ore, USA, 2002.

[19] J. B. Gray, T. R. Wentworth, and C. Brownie, "Extinction, colonization, and persistence of rare vascular flora in the longleaf pine-wiregrass ecosystem: responses to fire frequency and population size," Natural Areas Journal, vol. 23, no. 3, pp. 210-219, 2003.

[20] S. Poppinga, S. Hartmeyer, T. Masselter, I. Hartmeyer, and T. Speck, "Trap diversity and evolution in the family Droseraceae," Plant Signaling \& Behavior, vol. 8, no. 7, Article ID e24685, 2013.

[21] M. Méndez and P. S. Karlsson, "Costs and benefits of carnivory in plants: insights from the photosynthetic performance of four carnivorous plants in a subarctic environment," Oikos, vol. 86, no. 1, pp. 105-112, 1999.

[22] A. Pavloviĉ, L. Singerová, V. Demko, and J. Hudák, "Feeding enhances photosynthetic efficiency in the carnivorous pitcher plant Nepenthes talangensis," Annals of Botany, vol. 104, no. 2, pp. 307-314, 2009.

[23] V. A. Albert, S. E. Williams, and M. W. Chase, "Carnivorous plants: phylogeny and structural evolution," Science, vol. 257, no. 5076, pp. 1491-1495, 1992.

[24] A. M. Ellison and N. J. Gotelli, "Energetics and the evolution of carnivorous plants-Darwin's most wonderful plants in the world," Journal of Experimental Botany, vol. 60, no. 1, pp. 19-42, 2009.

[25] L. Adamec, "Mineral nutrition of carnivorous plants: a review," Botanical Review, vol. 63, no. 3, pp. 273-299, 1997. 
[26] J. J. Hutchens and J. O. Luken Jr., "Prey capture in the Venus flytrap: collection or selection?” Botany, vol. 87, no. 10, pp. 10071010, 2009.

[27] E. D. Schulze, G. Gebauer, W. Schulze, and J. S. Pate, "The utilization of nitrogen from insect capture by different growth forms of Drosera from Southwest Australia," Oecologia, vol. 87, no. 2, pp. 240-246, 1991.

[28] K. Kim and G. Yang, "Micro-propagation of Venus flytrap by shoot culture," Plant Cell, Tissue and Organ Culture, vol. 72, no. 1, pp. 95-98, 2003.

[29] R Development Core Team, R: A Language and Environment for Statistical Computing, R Foundation for Statistical Computing, Vienna, Austria, 2008.

[30] H. M. Hanslin and P. S. Karlsson, "Nitrogen uptake from prey and substrate as affected by prey capture level and plant reproductive status in four carnivorous plant species," Oecologia, vol. 106, no. 3, pp. 370-375, 1996.

[31] B. Juniper, R. Robins, and D. Joel, The Carnivorous Plants, London Academic Press, 1989.

[32] T. Gibson, "Differential escape of insects from carnivorous plant traps," American Midland Naturalist, vol. 125, no. 1, pp. 55-62, 1991. 

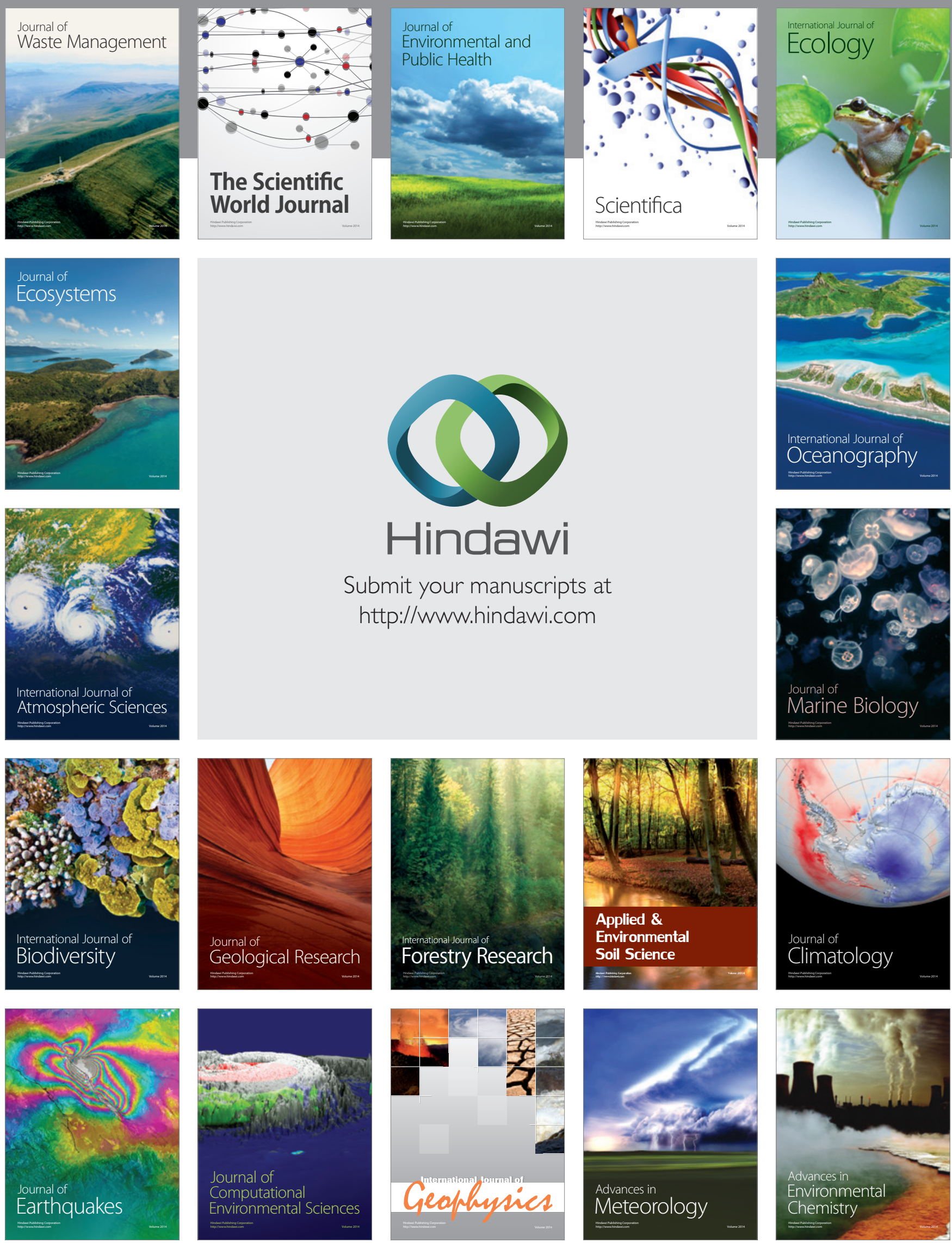